\title{
Fetal Intestinal Obstruction Induces Alteration of Enteric Nervous System Development in Human Intestinal Atresia
}

\author{
NAZIHA KHEN, FRANCIS JAUBERT, FREDERIQUE SAUVAT, LAURENT FOURCADE, \\ DOMINIQUE JAN, JELENA MARTINOVIC, MICHEL VEKEMANS, PAUL LANDAIS, \\ NICOLE BROUSSE, MICHELE LEBORGNE, CLAIRE NIHOUL-FÉKÉTÉ, \\ NADINE CERF-BENSUSSAN, SABINE SARNACKI, AND THE GROUP FOR THE STUDY OF \\ INTESTINAL ATRESIA
}

\begin{abstract}
INSERM E-0212 [N.K., N.C.-B., S.S.], Faculty Necker, 75743 Paris Cedex 15, France, Department of Pathology [F.J., N.B.], Department of Pediatric Surgery [F.S., L.F., D.J., C.N.-F., S.S.], Department of Histoembryology and Cytogenetic [J.M., M.V.], Department of Biostatistics [P.L.], Hôpital Necker
\end{abstract}

Enfants-Malades, 75730 Paris Cedex 15, France

\begin{abstract}
Intestinal motility disorders are a major cause of morbidity after surgical repair of intestinal atresia of unknown mechanism. We hypothesized that interruption of antenatal peristalsis may disturb the normal development of the enteric nervous system. Using a series of neuronal (synaptophysin, neuronal nitric oxide synthase, neurofilaments) and nonneuronal markers (glial acidic fibrillary protein and c-Kit) and immunohistochemistry, we have defined developmental steps of the enteric nervous system in normal intestine (12 fetuses, 15 children, and 4 adults) and their alterations above and below the obstacle in 22 human intestinal atresia compared with age-matched controls. Antisynaptophysin antibody revealed the progressive conversion of the myenteric plexus from a continuous belt into regularly spaced ganglions during normal fetal gut development and, by contrast, the significantly delayed appearance of individual neuronal ganglions in the distal segments of atresia $(p<0.05)$. Staging using three other markers for neuronal (neurofilaments and neuronal nitric
\end{abstract}

\section{ABSTRACT}

oxide synthase) and nonneuronal cells (glial acidic fibrillary protein) confirmed that maturation of the myenteric plexus was significantly delayed below atresia $(p<0.01)$. These results indicate that intestinal atresia impairs the development of the enteric nervous system and provide an anatomical substrate for the motility disorders observed after surgical repair. They point to the role of peristalsis in normal gut development and suggest that stimulation of peristalsis might be used to accelerate recovery. (Pediatr Res 56: 975-980, 2004)
ENS, enteric nervous system
Abbreviations
GFAP, glial fibrillary acidic protein
ICC, interstitial cells of Cajal
NF, neurofilament
nNOS, neuronal nitric oxide synthase
SIS, staining index for synaptophysin

Intestinal atresia is a common gut congenital disease with a prevalence of 2.8 for 10,000 births (1), detected by prenatal ultrasonography in the second or third trimester and confirmed at birth by intestinal obstruction. Treatment must be performed

Received December 12, 2003; accepted July 1, 2004

Correspondence: Sabine Sarnacki, M.D., Ph.D., Department of Pediatric Surgery, Hôpital Necker Enfants Malades, 149 rue de Sèvres, 75743 Paris Cedex 15, France; e-mail: sabine.sarnacki@nck.ap-hop-paris.fr

Supported by the Fondation de l'Avenir, the Association pour l'Etude de la Pathologie Pédiatrique, and the Fondation NRJ-Institut de France.

The Group for the Study of Intestinal Atresia from the Departments of Pediatric Surgery and Pathology of Hôpital Edouard Herriot, Lyon (J.P. Chappuis, R. Bouvier); Hôpital d'Enfants-La Timone, Marseille (J.M. Guys, J.F. Pellissier); Hôpital Gatien de Clocheville, Tours (H. Lardy, F. Fetissof); Hôpital Pontchaillou, Rennes (O. Azzis, M.P. Ramée); Hôpital Mère-Enfants, Nantes (G. Podevin, C. Laboisse); Hôpital Robert Debré, Paris (Y. Aigrain, M. Peuchmaur).

DOI: 10.1203/01.PDR.0000145294.11800.71 just after birth and consists of surgical resection of the atretic segment followed by primary anastomosis. Operative techniques and postoperative parenteral nutrition have improved the outcome, but prolonged intestinal motility disorders remain common in the postoperative period, even in the absence of any mechanical lesions (2). Although a dysfunction of the ENS has been suggested, the physiopathology of the motility disorders remains unknown $(3,4)$.

ENS comprises two main plexuses and schematically it can be assumed that the myenteric or Auerbach's plexus plays a major role in the regulation of motility and that the submucous or Meissner's plexus ensures regulation of intestinal secretion/ absorption $(5,6)$. Intestinal motility appears early at $16 \mathrm{wk}$ gestation (7) in fetal life and likely supports the nutritional function of the gut before birth $(8,9)$. Intestinal atresia, by 
interrupting antenatal gut peristalsis, may hamper the normal development of ENS. The few available experimental studies mainly focused on the dilated segment above atresia and showed hyperplasia of neuronal cells with decreased acetylcholinesterase activity (10). Only one study in a lamb model reported a lack of contractile activity in the distal segment (11). In humans, one study on a small number of patients and without developmental controls suggested reduced distribution of intramural nerves and pacemaker cells in proximal segments but failed to find any abnormality in the distal segment (3).

In this study, a panel of immunohistochemical markers for enteric neuronal and glial cells, as well as ICC, was used first to describe the development of ENS throughout fetal life to childhood and then to study the proximal and distal segments harvested during neonatal surgery for intestinal atresia. This comparison allowed us to demonstrate that intestinal atresia leads to impaired development of ENS not only above but also below the obstacle, the distal segment appearing less mature than the proximal one.

\section{MATERIALS AND METHODS}

Patients and tissue collection. A retrospective review of patients treated for an isolated form of small bowel atresia in six French centers over a 10-y period (1990-2000) was undertaken. Informed consent was obtained from parents of all participants. Patients with apple peel syndrome, familial or sporadic multiple atresia, or presenting with other abdominal pathologies such as gastroschisis, Hirschsprung disease, or cystic fibrosis were excluded. Among 123 patients born with small bowel atresia, 43 had isolated atresia but only 22 had available proximal and distal intestine to study. They were 12 boys and 10 girls with a median gestational age of $37 \mathrm{wk}$ (range, 34-40 wk). The level of atresia was jejunal in 12 cases and ileal in 10 .

Thirty-one normal intestinal specimens of different ages were studied as controls. Twelve fetal intestines from 8 to 35 wk gestation $(8,10,11,13,15,18,21,25,30,32,34$, and 35 wk) were obtained from medical elective termination of pregnancies with the permission of the local ethical committee of Hôpital Necker Enfants-Malades. They were from jejunal and ileal portions of the gut. Intestinal specimens from 12 infants aged from $1 \mathrm{~d}$ to 8 mo and from three children 4,7 , and $9 \mathrm{y}$ old were also collected. They were taken from normal margins of resected Meckel's diverticulum in nine cases and intestinal duplications in six. Four additional specimens consisted of normal adult intestine $(35,39,53$, and 92 y old) resected during restorative surgery for abdominal cancer.

Immunohistochemistry. All tissue samples were fixed by immersion in $10 \%$ formalin and embedded in paraffin, a technique that minimizes tissue deformation. The following antibodies were used: antisynaptophysin (1:20, rabbit polyclonal, DakoCytomation, Trappes, France), anti-NF (1:50, mouse monoclonal IgG1, DakoCytomation), -nNOS (1:400, rabbit polyclonal, Santa Cruz Biotechnology, Tebu Bio, Le Perray, France), -GFAP (1:250, mouse monoclonal IgG1, DakoCytomation), and -c-Kit (1:400, rabbit polyclonal, DakoCytomation). Circular sections of $5-\mu \mathrm{m}$ thickness were prepared deparaffinized in xylene and antigen retrieval by microwave oven heating in citric buffer pH6 was performed. Sections were successively incubated with primary antibodies, biotinylated anti-mouse or anti-rabbit immunoglobulin secondary antibody (Detection kit Dako ChemMate), and streptavidin-conjugated horseradish peroxidase (Detection kit Dako ChemMate) for 30 min, respectively. Between each step, sections were washed in Tris-HCL buffer. Staining was revealed by a 6-min incubation in 3,3'-diaminobenzidine tetrachloride (Detection kit Dako ChemMate) and $\mathrm{H}_{2} \mathrm{O}_{2}$ and nuclei were counterstained by Harris' hematoxylin. Proximal and distal bowel segments from a given patient were always stained during the same procedure. Negative controls (control mouse isotype or rabbit serum) did not show any staining except for endogenous peroxidase activity of eosinophils.

Quantification. Slides were analyzed by light microscopy. Morphometric measurements of the myenteric plexus were performed using synaptophysin. This protein, located in small synaptic vesicles, is a reliable marker of neuronal cells in the central (12) and peripheral (13) nervous system and provided early and specific staining of the myenteric plexus from $8 \mathrm{wk}$ of gestation to the adult period. To quantify the progressive conversion of the myenteric plexus from a continuous belt into regularly spaced knots throughout development, we defined a SIS as the ratio between total length of stained ganglia and intestinal length of the specimen, measured on the line drawn by the myenteric plexus on transversal sections. Measurements were performed on digital recorded images using the SigmaScan Pro 5.0 software (SPSS Science, Chicago, IL).

Two independent investigators (N.K, F.J.) evaluated staining with the other ENS markers, semi-quantitatively and blindly. The staging system was established as follows. A grid was established after a first screening of all specimens for each marker. Four stages of development $(0-3)$ were defined for each marker according to the type of staining. For nNOS and NF, stages were defined according to the size and number of stained neurons in ganglia of myenteric plexus. We observed four types of labeling: no staining, staining of small neurons (immature), staining of large neurons (mature). In this latter group, we observed two main subtypes defined by the percentage of labeled neurons (over or under $50 \%$ ). The order of numbering was then given logically according to the successive appearance of the four stages in controls. Staining in intestinal atresia was further scored according to the previously defined scale. For GFAP and c-Kit, we followed the same procedure to define the stages but the criteria were based only on the number of stained cells per ganglia.

Statistical analysis. Data were statistically analyzed using Statistica 6.0 software (StatSoft, Tulsa, OK). Age-dependent variation of SIS in controls was calculated with a simple regression analysis. Results between proximal and distal segments of atresia were compared using a Wilcoxon pairmatched test. A $p$ value $<0.05$ was considered significant.

\section{RESULTS}

Study of neuronal markers. The pattern of expression of nNOS and NF was comparable in controls during development 
and was scored on the basis of the size and number of stained neurons in ganglia of the myenteric plexus, as follows: stage 0: no staining; stages 1-3: intracellular staining of small neuronal cells only (stage 1), of $>50 \%$ large neuronal cells per ganglia (stage 2), and of $<50 \%$ large neuronal cells per ganglia (stage 3). For both markers, stained cells were first detected at approximately $14 \mathrm{wk}$ gestation ( $13 \mathrm{wk}$ for nNOS and $15 \mathrm{wk}$ for NF) and staining remained at stage 1 until birth. Large stained neurons (stage 2) were first seen in the neonatal period. The mature stage 3 was reached at 3 and 5 mo for NF and nNOS, respectively, and remained unchanged until the adult age (Fig. $1 A$, panels $a$ and $c$; Fig. $1 B$, panels $a, b, e$, and $f$ ). In intestinal atresia, scores were statistically different in proximal and distal segments in 19/22 (86\%) for nNOS $(p<0.005)$ and $15 / 22$ $(68 \%)$ for NF $(p=0.005)$. The score in the proximal segment at birth was stage 2 , a stage observed in controls in the neonatal period in 10/22 (45\%) and 14/22 (64\%) specimens for nNOS and NF, respectively, and stage 3 in 12/22 (55\%) cases for nNOS and 8/22 cases (36\%) for NF. In contrast, 11/22 (50\%) specimens taken from distal segments were stage 1, a stage observed in control fetuses, for both NF and nNOS. The other samples were stage 2 (Fig. $1 A$, panels $b$ and $d$; Fig. $1 B$, panels $c, d, g$, and $h$ ).

During the antenatal period, the staining index of the myenteric plexus with antisynaptophysin antibody (SIS) decreased regularly. Its value of $80 \%$ at $10 \mathrm{wk}$ gestation reflected the dense aspect of the myenteric plexus. Its decrease to $30 \%$ at $34 \mathrm{wk}$ gestation was due to the conversion of the myenteric plexus from a continuous belt into regularly spaced knots. After birth, the SIS slowly increased to $50 \%$ until $5 \mathrm{mo}$, then decreased again and remained around $30 \%$. A significant negative correlation between SIS and age of controls was found $(p$ $<0.001$ ) between 8 wk of gestation and 8 mo after birth (Fig. $2 A$, panel $a$; Fig. $2 B$, panels $a$ and $b$ ). The SIS were calculated for the 18/22 specimens of intestinal atresia, in which more than half the intestinal circumference was available for analysis in both the proximal and distal segments. The median and range of the SIS in proximal segments were 23\% (12-34\%) and thus lower than the SIS calculated for all normal controls, whatever the age of observation. In contrast, the median and range of the SIS in distal segments were $43 \%$ and $45 \%$ (33-66\%), respectively, values observed in control fetuses between 25 and $35 \mathrm{wk}$ gestation. Results between proximal and distal segments were significantly different $(p<0.001)$ (Fig. $2 A$, panel $b$; Fig. $2 B$, panels $c$ and $d$ ).

Altogether, results obtained with neuronal markers point to the asynchronic development of the myenteric plexus, which appears delayed below the obstacle and accelerated above.

Study of glial cells. The pattern of expression of GFAP was scored on the basis of the mean number of stained cells per ganglia in the myenteric plexus as follows: stage 0: no staining; stage 1: less than one cell stained per ganglia; stage 2: one to two cells stained per ganglia; stage 3: more than two stained cells per ganglia. All control specimens were stage 0 before 30 wk of gestation, stage 1 appearing at $30 \mathrm{wk}$ gestation, and stage 2 during the perinatal period. Stage 3 was reached at 2 mo of life and remained unchanged until the adult period (Fig. 3A, panel $a$ ). In intestinal atresia, results were statistically different
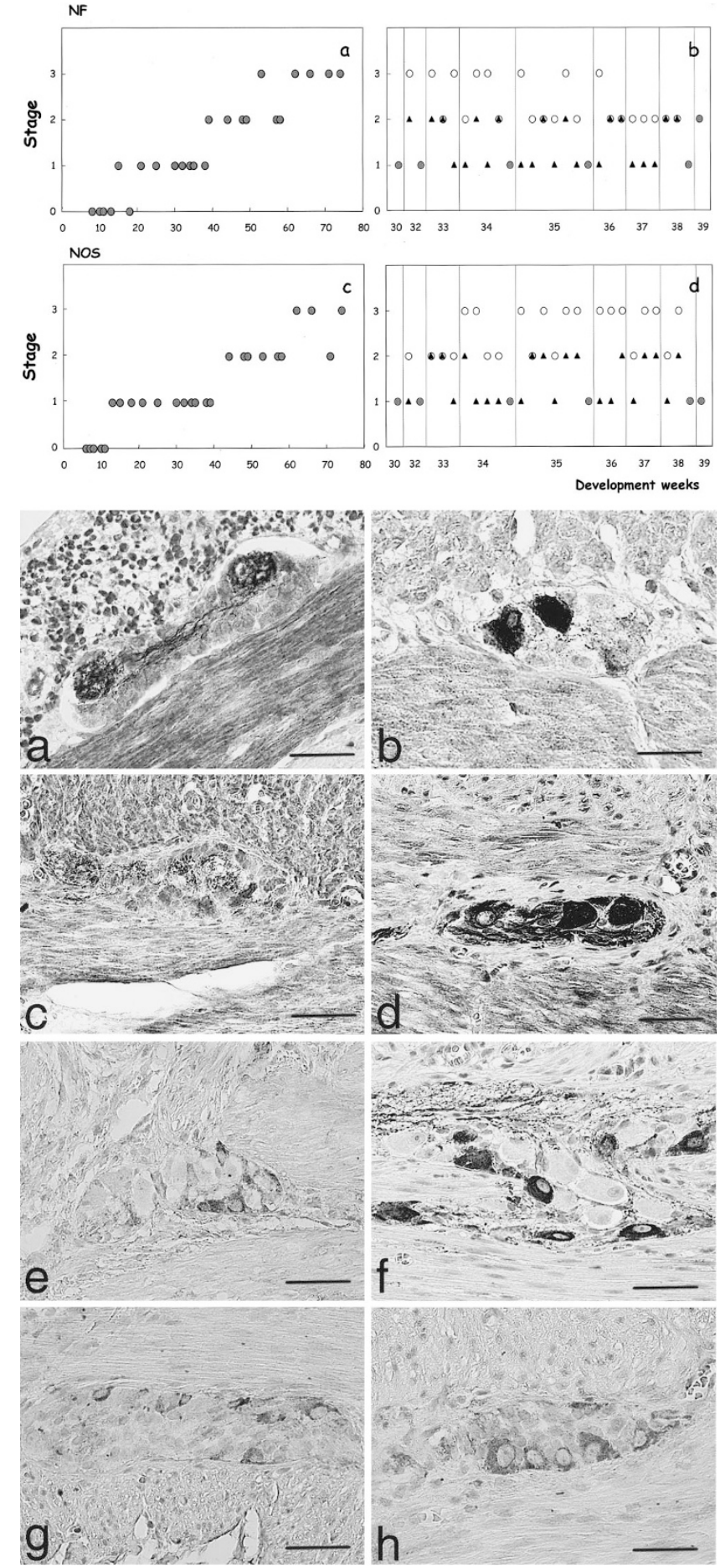

Figure 1. Neuronal markers (NF and nNOS). (A) Graph showing stages of myenteric plexus development using anti-NF $(a, b)$ and anti-nNOS $(c, d)$ antibodies graded as described in "Materials and Methods": controls ( $a, c$, gray circles), proximal (white circles) and distal (black triangles) segments of intestinal atresia $(b, d)$. Panels $a$ and $c$ show staging in controls according to the gestational age added or not with age of life, expressed in weeks after birth. Panels $b$ and $d$ show staging in intestinal atresia according to gestational age at surgery. To allow an easier comparison, values in age-matched controls have been indicated in these latter panels. $(B)$ Immunohistochemical expression of NF $(c)$ and nNOS $(g)$ in distal segment of a 39-wk-old patient with atresia is similar to that observed in a 32-wk control fetal gut $(a, e)$ with rare positive cells of small size (stage 1). In contrast, the proximal segment of the same patient $(d, h)$ is similar to that observed after birth in a 4-y-old control $(b, f)$ with numerous positive large cells showing an abundant positive cytoplasm and a large nucleus (stage 3 ). All specimens are taken from ileum (original magnifications: $\times 200$; scale bar 50 microns). 

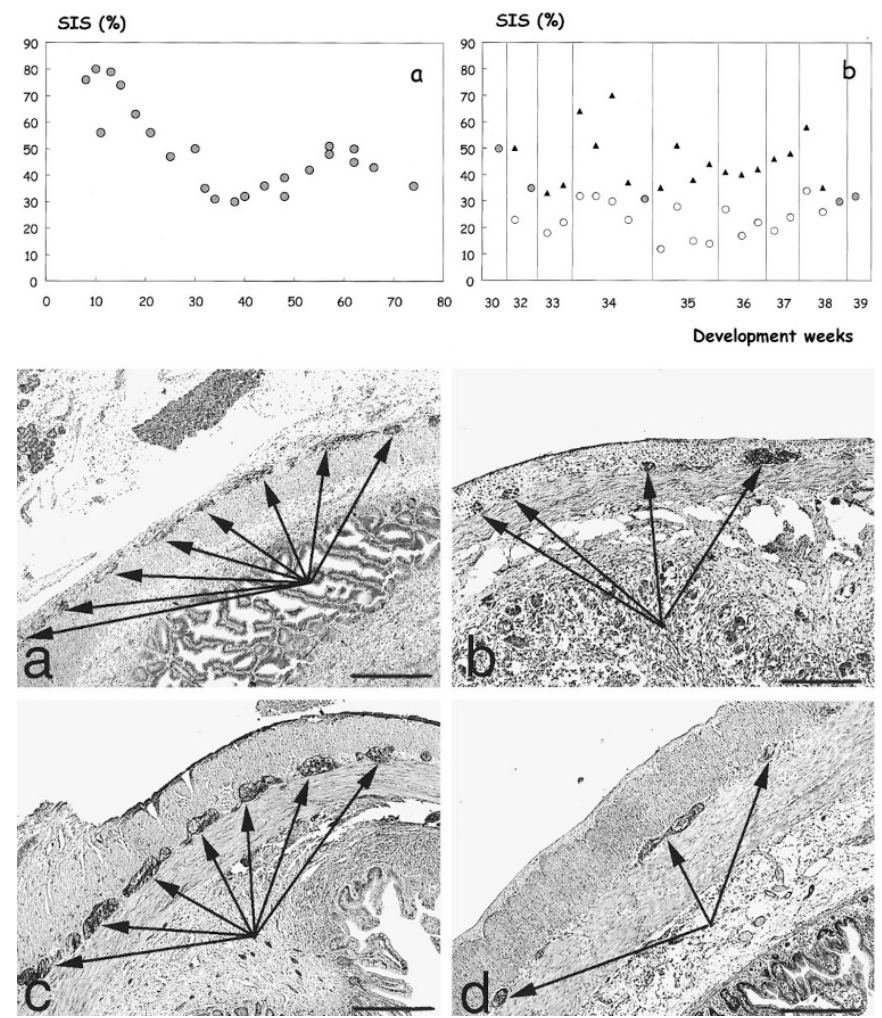

Figure 2. Staining index for synaptophysin. (A) Graph showing the progressive conversion of the myenteric plexus from a continuous layer into regularly spaced knots was quantified using SIS, defined as the ratio between the stained ganlia length and total intestinal length on the same specimen measurement being done on a line drawn through the myenteric plexus. The SIS is shown according to the gestational age added or not with age of life in weeks after birth, either in controls (panel a, gray circles) or in proximal (white circles) and distal (black triangles) segments of intestinal atresia (panel b). To allow an easier comparison with controls, values in age-matched controls have been indicated in this latter panel. (B) Immunohistochemical staining by synaptophysin shows that ENS organization is similar in atresia of a 36-wk-old patient (c) and that of a 15-wk-old fetus (a) with large ganglia forming an almost continuous layer. By contrast, the pattern observed in atresia proximal segment $(d)$ is comparable to that of a control at birth $(b)$ with spaced small ganglia. All specimens are obtained from ileum (original magnifications: $a \times 100 ; b, c, d$ $\times 25$; scale bar 500 microns).

between proximal and distal segments $(p<0.001)$ and underlined the distinct kinetics of maturation of the myenteric plexus between both segments. Thus, proximal segments were stage 3 in 16/22 cases (73\%), and, among them, 11 showed a staining stronger than the one observed in controls, whatever the age of observation. In contrast, distal segments were stage 0 in $11 / 22$ cases $(50 \%)$ and stage 1 in $5 / 22$ cases (23\%). Only six cases were stage $2(n=3)$ or stage $3(n=3)$ on the distal segment and in these latter cases, the corresponding proximal segment was either stage 2 or 3 (Fig. 3A, panel $b$; Fig. 3B, panels $a$ and $b$ ).

Study of ICC. The expression of c-Kit was scored on ICC located around the myenteric plexus as follows: stage 0: no staining; stage 1: a few cells stained; stage 2: scattered staining; stage 3: continuous staining delineating the myenteric plexus. Control specimens $<13$ wk of gestation were stage 0 . Stage 1 appeared at $13 \mathrm{wk}$ gestation, stage 2 at $20 \mathrm{wk}$ gestation, and stage 3 was only seen in the postnatal period (Fig. 3A, panel $c$ ). In intestinal atresia, scores were statistically different between
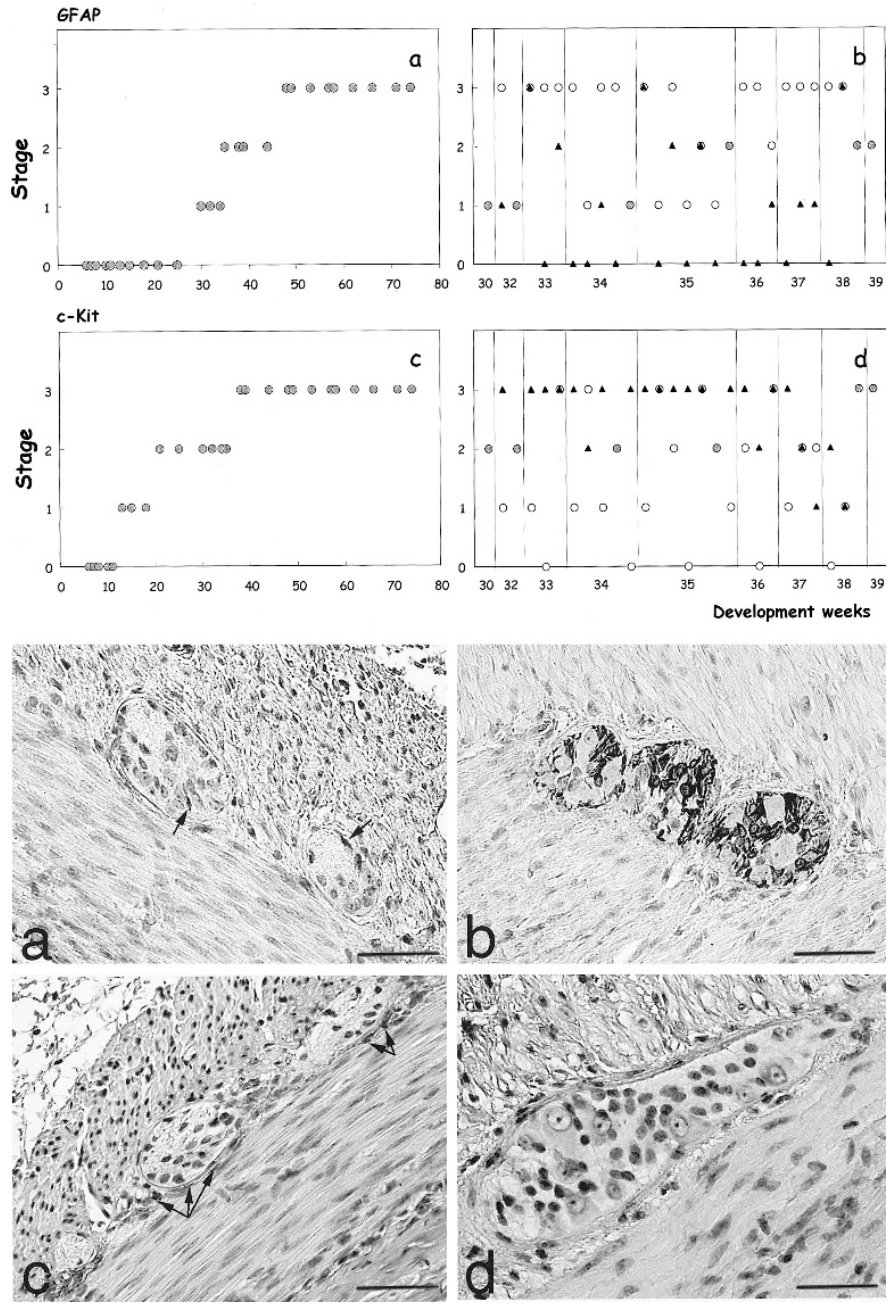

Figure 3. Nonneuronal markers (GFAP and c-Kit). (A) Graph showing the stages of the myenteric plexus development with anti-GFAP $(a, b)$ and anti-c-Kit $(c, d)$ antibodies was obtained as described in "Materials and Methods," in controls (a, c, gray circles), proximal (white circles) and distal (black triangles) segments of intestinal atresia $(b, d)$. Panels $a$ and $c$ show staging in controls according to the gestational age added or not with age of life, expressed in weeks after birth. Panels $b$ and $d$ show staging in intestinal atresia according to gestational age at surgery. To allow an easier comparison with controls, values in age-matched controls have been indicated in these latter panels. $(B)$ Immunohistochemistry of atresia distal segment in a 39-wkold baby ( $a$ ) shows a GFAP staining comparable to that observed before $30 \mathrm{wk}$ in controls with scarce GFAP-positive glial cell (arrow). In marked contrast, the anti-GFAP antibody labels numerous positive cells in the proximal segment $(b)$, as only observed after 2 mo in control children (stage 3). C-Kitpositive cells form a continuous thin line around the myenteric ganglia (stage 3 ) in the distal segment of atresia (c). In contrast, no positive cells (stage 0 ) can be evidenced in the proximal segment $(d)$. All specimens are derived from ileum (original magnifications: $\times 200$; scale bar 50 microns).

proximal and distal segments $(p=0.001)$. Distal segments were stage 3 in 16/22 cases (73\%) and thus comparable to neonatal controls. In contrast, in the proximal segment, only five cases were scored stage 3 whereas stage 0 or 1 was observed in 13/22 cases (59\%) and stage 2 in 4/22 (18\%) (Fig. $3 A$, panel $d$; Fig. $3 B$, panels $c$ and $d$ ).

\section{DISCUSSION}

Using a panel of neuronal and nonneuronal markers revealed by immunohistochemistry, we provide the first detailed study 
of the development of the ENS in humans from the 8th wk of gestation until adult age. Furthermore, our work points to obvious alterations of ENS development in neonates with intestinal atresia and provides an anatomical substrate for the motility disorders observed after surgical repair.

The five markers used to study the different components of the ENS allowed delineation of several developmental steps. The most informative marker was synaptophysin, an integral membrane protein of the small synaptic vesicles, which permitted quantitative follow-up on the progress of the myenteric plexus from a continuous belt at the embryonic stage into distinct and regularly spaced ganglia during fetal life. Progressive maturation of ENS was further evidenced using antibody recognizing an epitope common to the three subnunits of the cytoskeletal neuronal protein NF and nNOS, a nonadrenergic noncholinergic mediator of the nervous system produced by enteric neurons with inhibitory functions $(14,15)$. The latter protein plays a key role in the regulation of intestinal motility $(16,17)$. Both markers had a similar pattern of expression during development, being first detected at the beginning of the second trimester. Positive cells increased in size during the perinatal period and represented the main population of neurons until 5 mo. Their proportion among neurons decreased thereafter to become minor during childhood, a finding consistent with previous observations by Eaker et al. (18) in the postnatal period in rats using a panel of anti-NF antibody. Using NADPH diaphorase histochemistry, previously used for the indirect labeling of NO synthesizing cells, Brandt et al. (19) also described a decrease in the density of stained neurons during the human fetal development, resulting in increased interspacing between myenteric ganglia. After birth, the numbers of NADPH diaphorase-positive enteric neurons was shown to keep on decreasing during the first 3 y (20).

Two other markers, GFAP (21) and c-Kit, were useful to follow up the development of two important nonneuronal cell types in the myenteric system: glial cells and ICC, respectively. Cajal cells viewed as intestinal pacemaker cells are mandatory for intestinal motility, as shown in mice invalidated for the c-kit gene (22). Glial cells, like enteric neuronal cells, originate from the neural crest and probably share the same precursor (23). Recent work in the CNS suggests that, besides providing nutritional support, glial cells participate in the regulation of neuronal differentiation and in the control of synaptic connection (24). In controls, GFAP staining was absent until $30 \mathrm{wk}$ gestation and weak during the last part of the gestation, the number of stained cells increasing during the first 2 mo after birth. C-Kit staining of ICC was visible at $13 \mathrm{wk}$, first as a discontinuous line around the myenteric plexus, which became continuous on and after $18 \mathrm{wk}$, findings similar to those reported by Wester et al. (25) and Kenny et al. (26). After birth, c-Kit staining appeared as a thin line surrounding the plexus. Altogether, these markers allowed one to draw a precise pattern of ENS development, which gives an anatomical substrate to the progressive acquisition and maturation of intestinal motility both during the ante and postnatal period (27). This first extensive description of ENS development from fetal life to adult stage provides a basis for classifying the still-undefined pediatric gut motility disorders. It allowed us to unambiguously demonstrate the abnormal maturation of ENS in patients with atresia compared with age-matched controls and its lack of synchronization between proximal and distal segments.

Thus, in most patients with intestinal atresia, markers for neuronal and glial cells showed striking differences above and below atresia, suggestive of accelerated maturation in the proximal segment and delayed maturation in the distal one. First, the median of the SIS in the proximal segment was less than in gestational age-matched controls. Decreased density in the myenteric plexus in the proximal segment was also suggested by previous reports using the neuronal marker PGP 9.5 (3) or a two-dimensional analysis of the plexus distribution (4). Such a result cannot be ascribed only to distension above obstacle since, in the majority of the patients, the number of glial cells was increased compared with gestational agematched controls and similar to that observed after 2 mo of life. The differences were even more striking when results were compared with those in the distal segment. SIS was increased in the distal segment, reflecting a more continuous aspect of the myenteric plexus similar to that observed in fetuses between 25 and 35 wk of gestation. Staging using NF and nNOS was less than expected for age in $50 \%$ of cases and less than in the proximal segment in most patients. Accordingly, GFAP staining of glial cells was undetectable in the distal segment in 50\% of patients and weak in $22 \%$, suggesting a profound perturbation in the maturation of glial cells below the obstacle. In contrast, overexpression of GFAP was noted in the proximal segments in $50 \%$ of patients.

Significant differences in c-Kit staining between the proximal and distal segments were also observed in intestinal atresia. Thus, c-Kit staining was normal for age in the distal segment but markedly decreased above the obstacle in $>60 \%$ of patients, in agreement with previous findings by Masumoto et al. (3). Diminution of c-Kit staining was observed in adult rats above the obstacle 18-24 d after experimental obstruction (28), suggesting that loss of c-Kit staining and ICC cells was secondary to intestinal struggle against obstruction.

These results indicate that gut dysmotility after surgical repair of intestinal atresia is not only due to the damage of the proximal dilated segment but is also the consequence of a distinct pathology of the distal segment. Previous studies in intestinal atresia focused on the dilated proximal segment, similar to what happened in initial studies in Hirschsprung's disease. To our knowledge, only one experimental study of fetal intestinal obstruction in sheep provided evidence of functional alterations in the distal segment. Based on the recording of myoelectric activity, the authors reported an absence of spike potentials in the distal segment (11). Our results provide an anatomical basis for this functional finding, as they show a defect in the maturation of the myenteric plexus below the obstacle. Using SIS as a criteria of maturation of the ENS, we recently observed similar findings in an experimental rat model of fetal intestinal obstruction with an immature aspect of the distal segment, when compared with the proximal one or two normal age-matched controls (unpublished data). This defect might be related to interruption of either rostro-caudal signaling requested in many development processes and/or of me- 
chanical stimuli provided by the peristalsis, which might be mandatory for full maturation of ENS (29). This latter hypothesis is sustained by the recent work of Farge (30), which showed that mechanical stresses can affect the expression of developmental genes, providing evidence that molecular signals are not the only forces that pattern and shape the developing embryo. Physical forces have also been proposed as playing a role in remodeling the lung during development by modulating apoptosis (31). The impaired maturation of the distal segment observed in our work provides a rationale for the persistence of motility disorders despite the treatment of the proximal segment in many cases. To shorten the postoperative recovery period, we propose mechanical stimulation of the distal segment by minimal enteral feeding as performed in preterm infants (32) together with retrograde enema or anterograde enema via a stoma. The benefit of such an approach might be ascertained by correlating immunohistochemical findings with the clinical course. This question will be addressed in a prospective French cooperative prospective study. Finally, our findings suggest that function and development are closely related and point to the fact that congenital defects entail complex sequences of epigenetic events that must be taken into account in analysis and treatment.

Acknowledgments. The authors thank G. Pivert for technical assistance and T. Attié for helpful discussion.

\section{REFERENCES}

1. Cragan JD, Martin ML, Moore CA, Khoury MJ 1993 Descriptive epidemiology of small intestinal atresia, Atlanta, Georgia. Teratology 48:441-450

2. Dalla Vecchia LK, Grosfeld JL, West KW, Rescorla FJ, Scherer LR, Engum SA 1998 Intestinal atresia and stenosis: a 25-year experience with 277 cases. Arch Surg 133:490-496

3. Masumoto K, Suita S, Nada O, Taguchi T, Guo R, Yamanouchi T 1999 Alterations of the intramural nervous distributions in a chick intestinal atresia model. Pediatr Res 45:30-37

4. Watanabe Y, Ando H, Seo T, Katsuno S, Marui Y, Horisawa M 2001 Twodimensional alterations of myenteric plexus in jejunoileal atresia. J Pediatr Surg 36:474-478

5. Goyal RK, Hirano I 1996 The enteric nervous system. N Engl J Med 334:1106-1115

6. Gershon MD, Chalazonitis A, Rothman TP 1993 From neural crest to bowel: development of the enteric nervous system. J Neurobiol 24:199-214

7. Grand RJ, Watkins JB, Torti FM 1976 Development of the human gastrointestinal tract. A review. Gastroenterology 70:790-810
8. Trahair JF 1993 Is fetal enteral nutrition important for normal gastrointestinal growth?: a discussion. JPEN J Parenter Enteral Nutr 17:82-85

9. Surana R, Puri P 1994 Small intestinal atresia: effect on fetal nutrition. J Pediatr Surg 29:1250-1252

10. Tepas JJ, Wyllie RG, Shermeta DW, Inon AE, Pickard LR, Haller Jr JA 1979 Comparison of histochemical studies of intestinal atresia in the human newborn and fetal lamb. J Pediatr Surg 14:376-380

11. Doolin EJ, Ormsbee HS, Hill JL 1987 Motility abnormality in intestinal atresia. J Pediatr Surg 22:320-324

12. Grunnet ML 1995 A lectin and synaptophysin study of developing brain. Pediatr Neurol 13:157-160

13. Hitchcock RJ, Pemble MJ, Bishop AE, Spitz L, Polak JM 1992 Quantitative study of the development and maturation of human oesophageal innervation. J Anat 180:175183

14. Bredt DS, Hwang PM, Snyder SH 1990 Localization of nitric oxide synthase indicating a neural role for nitric oxide. Nature 347:768-770

15. Bult H, Boeckxstaens GE, Pelckmans PA, Jordaens FH, Van Maercke YM, Herman AG 1990 Nitric oxide as an inhibitory non-adrenergic non-cholinergic neurotransmitter. Nature 345:346-347

16. Vanderwinden JM, De Laet MH, Schiffmann SN, Mailleux P, Lowenstein CJ, Snyder SH, Vanderhaeghen JJ 1993 Nitric oxide synthase distribution in the enteric nervous system of Hirschsprung's disease. Gastroenterology 105:969-973

17. Timmermans JP, Barbiers M, Scheuermann DW, Bogers JJ, Adriaensen D, Fekete E, Mayer B, Van Marck EA, De Groodt-Lasseel MH 1994 Nitric oxide synthase immunoreactivity in the enteric nervous system of the developing human digestive tract. Cell Tissue Res 275:235-245

18. Eaker EY, Sallustio JE, Harris JM, Shaw G 1993 Myenteric plexus neurons have developmentally acquired differences in the medium molecular weight subunit of neurofilament protein. Neuroscience 53:561-570

19. Brandt CT, Graham A, Tam PK 1997 Densities of nitric oxide synthesizing nerves in smooth muscles of human gut during fetal development. J Pediatr Surg 32:1314-1317

20. Wester T, O'Briain DS, Puri P 1999 Notable postnatal alterations in the myenteric plexus of normal human bowel. Gut 44:666-674

21. Jessen KR, Mirsky R 1980 Glial cells in the enteric nervous system contain glial fibrillary acidic protein. Nature 286:736-737

22. Huizinga JD, Thuneberg L, Kluppel M, Malysz J, Mikkelsen HB, Bernstein A 1995 $\mathrm{W} /$ kit gene required for interstitial cells of Cajal and for intestinal pacemaker activity. Nature 373:347-349

23. Burns AJ, Douarin NM 1998 The sacral neural crest contributes neurons and glia to the post-umbilical gut: spatiotemporal analysis of the development of the enteric nervous system. Development 125:4335-4347

24. Ullian EM, Sapperstein SK, Christopherson KS, Barres BA 2001 Control of synapse number by glia. Science 291:657-661

25. Wester T, Eriksson L, Olsson Y, Olsen L 1999 Interstitial cells of Cajal in the human fetal small bowel as shown by c-kit immunohistochemistry. Gut 44:65-71

26. Kenny SE, Connell G, Woodward MN, Lloyd DA, Gosden CM, Edgar DH, Vaillant C 1999 Ontogeny of interstitial cells of Cajal in the human intestine. J Pediatr Surg 34:1241-1247

27. Bisset WM, Watt JB, Rivers RP, Milla PJ 1988 Ontogeny of fasting small intestinal motor activity in the human infant. Gut 29:483-488

28. Ekblad E, Sjuve R, Arner A, Sundler F 1998 Enteric neuronal plasticity and a reduced number of interstitial cells of Cajal in hypertrophic rat ileum. Gut 42:836-844

29. Giaroni C, De Ponti F, Cosentino M, Lecchini S, Frigo G 1999 Plasticity in the enteric nervous system. Gastroenterology 117:1438-1458

30. Farge E 2003 Mechanical induction of Twist in the Drosophila foregut/stomodeal primordium. Curr Biol 13:1365-1377

31. Del Riccio V, van Tuyl M, Post M 2003 Apoptosis in lung development and neonatal lung injury. Pediatr Res 55:183-189

32. Berseth CL 1989 Gestational evolution of small intestine motility in preterm and term infants. J Pediatr 115:646-651 